\title{
Stability of A Class of Multi-Agent Tracking Systems with Unstable Subsystems
}

\author{
Lijing Dong, Senchun Chai, Baihai Zhang, Sing Kiong Nguang, and Al Savvaris
}

\begin{abstract}
In this work, we pre-deploy a large number of smart agents to monitor an area of interest. This area could be divided into many Voronoi cells by using the knowledge of Voronoi diagram and every Voronoi site agent is responsible for monitoring and tracking the target in its cell. Then, a cooperative relay tracking strategy is proposed such that during the tracking process, when a target enters a new Voronoi cell, this event triggers the switching of both tracking agents and communication topology. This is significantly different from the traditional switching topologies. In addition, during the tracking process, the topology and tracking agents switch, which may lead the tracking system to be stable or unstable. The system switches either among consecutive stable subsystems and consecutive unstable subsystems or between stable and unstable subsystems. The objective of this paper is to design a tracking strategy guaranteeing overall successful tracking despite the existence of unstable subsystems. We also address extended discussions on the case where the dynamics of agents are subject to disturbances and the disturbance attenuation level is achieved. Finally, the proposed tracking strategy is verified by a set of simulations.
\end{abstract}

Index Terms-tracking strategy, switched multi-agent systems, Voronoi diagram, unstable subsystems.

\section{INTRODUCTION}

Inspired by the group activities of humans and collective behaviour of insects or birds, researchers from various research fields have done a lot of work to explain this kind of phenomena [1, 2]. Multi-agent systems [3-5] are then developed and have been widely used in the applications of mobile robots [6] and unmanned vehicles [7, 8]. Tracking problem of a target is a typical issue of multi-agent systems and has drawn

L. Dong is with School of Mechanical, Electronic and Control Engineering, Beijing Jiaotong University, Beijing 100044, PR China.

S. Chai and B. Zhang are with School of Automation, Beijing Institute of Technology, Beijing 100081, PR China (Corresponding author: S. Chai, e-mail: chaisc97@bit.edu.cn).

S. K. Nguang is with the Department of Electrical and Computer Engineering, The University of Auckland, Private Bag, 92019 Auckland, New Zealand.

A. Savvaris is with School of Aerospace, Transport and Manufacturing, Cranfield University, Bedfordshire, MK43 0AL, United Kingdom. much attention of researchers [9-14]. In most of the existing literatures, the topologies of multi-agent systems are supposed to be fixed topologies or switching topologies. [10] study tracking problems for multi-agent systems under switching topologies, which are modelled by switching between several fixed topologies. In our previous works [11-14], we have studied the tracking problem for one target under a timevarying topology and in [14], the communication delay is also considered.

In [15], the authors propose a relay pursuit scheme to capture a maneuvering target on a plane, monitored by a group of agents. At each instant of time, only one pursuer is assigned the task of capturing the moving target. The plane is divided into a number of regions by the pursuers with knowledge of Voronoi diagram, a vigorous tool of dividing space [16-18]. In [15], the agents are governed by first-order integral kinematics and the target is tracked by a single pursuer without cooperation. As mentioned in the work of [15], in some situations, the capture is successful only if the pursuers cooperate with each other. In that sense, relay pursuit strategies are viewed as an intermediate option offering a simpler alternative for a multi-agent tracking problem involving multiple pursuers, whose solution is known to be very hard [19]. Another possible extension is to consider the case that agents are described by more realistic kinematics not just integral dynamics.

Motivated by the above discussion, in this paper, we consider a more complicated scenario where a certain area is monitored by a large number of mobile agents, described by nonlinear dynamics, when targets move into this area, a preset number of mobile agents cooperate to track the targets. In practice, our proposed scheme can be appropriately used for protecting sensitive areas against offensive intrusion [20] and occasionally tracking targets in a typical military, environmental, or habitat monitoring applications [21].

When the target successively transits from one Voronoi cell to another, the tracking agent with furthest distance to 
the target is replaced by the corresponding Voronoi cell agent. The switching of tracking agents thus results in tracking error jumps and involves switching of communication topologies, which is quite different with the traditional time-triggered switching topologies. The topology could be unconnected due to the fact that the topologies and tracking agents switch during the tracking process, which may lead the corresponding system to be unstable. The system not only switches among consecutive stable subsystems or consecutive unstable subsystems but also between stable and unstable subsystems, which is wellknown a difficult issue to tackle[22], [23]. In [24], the authors consider a setting that the weak connectivity of the interaction topologies is kept for some disconnected time intervals with short length. The authors in [25] also obtain some results on the case where the individual uncoupled system is allowed to be strictly unstable. In both of the two papers, the agents are assumed to be holding linear dynamics and the agents do not switch during the moving process.

The goal of this paper is to design a tracking strategy guaranteeing successful tracking of a class of nonlinear tracking system with tolerance of unstable subsystems. We also investigate the situation in which the dynamics of agents are subject to external disturbances. From a practical point of view, the external disturbances can not be avoided in the presence of communication noises and environmental effects [26, 27]. The effects of the disturbance on multi-agent systems have been investigated in [28-32]. In [30], a fuzzy $H \infty$ controller is designed to achieve a guaranteed $H \propto$ performance level for nonlinear multi-agent systems in which the nonlinear agents are described by Takagi-Sukeno (T-S) fuzzy models. [32] achieves the disturbance attenuation for multi-agent systems with switching topology networks with an average dwell time. Most of the existing literatures relevant to the disturbance attenuation issue for multi-agent systems are with fixed topology [28-31] or switching among stable subsystems [32]. The tracking problem for multi-agent systems with both unstable subsystems and disturbance has not been tackled in existing literatures, which motivates us to do this work.

The main contributions are stated as follows. Firstly, a new multi-agent tracking problem frame is constructed to simulate a realistic area-monitoring and target tracking situation, and a cooperative relay strategy is proposed to solve this problem. Secondly, we derive a new mathematical model for the tracking problem. Such a model can effectively solve the jumping problem of the tracking errors caused by the relay scheme. Thirdly, a new switched technique is adopted to tackle the existence of unstable subsystems, which makes the analysis of the tracking problem more difficult. Furthermore, we also discuss the case that the agents are constrained by external disturbances because such disturbances can not be avoided due to the presence of communication noises or environmental effects.

The following notations will be used throughout this paper. $\mathbb{N}$ is used to denote the set of nonnegative integers. $\mathbb{R}^{m}$ denotes the set of all $m$-dimensional real column vectors. For a real symmetric positive semi-definite matrix $P, \lambda_{i}(P)$ denotes its $i$-th eigenvalue. $\lambda_{\max }(P)$ and $\lambda_{\min }(P)$ represent the maximum and minimum eigenvalue of $P$, respectively. $\|\cdot\|$ stands for the Euclidean norm. $\mathscr{L}_{2}[0, \infty)$ denotes the space of square integrable vector functions over $[0, \infty)$, and for a time-varying vector $v$, the $\mathscr{L}_{2}$ norm is defined as $\|v\|_{\mathscr{L}_{2}}=\sqrt{\int_{0}^{\infty} v^{T}(t) v(t) d t}$.

\section{RELATED PRELIMINARIES}

\section{A. Voronoi diagrams}

The monitored 2-dimensional space is divided into a number of regions (Voronoi cells) with the assistance of Voronoi diagrams. A mathematical representation for Voronoi diagrams can be described as follows.

Let $X$ be a 2-dimensional space. There are $N_{a}$ agents distributed in the supervisory area of $X . a_{i}$ denotes the $i-$ th agent and $\mathcal{N}_{x}=\left\{a_{i}, 1 \leq i \leq N_{a}\right\} \subseteq X$ is the set of agents distributed on plane $X$. The Voronoi diagram is a partitioning of plane $X$ into regions based on distance to agents in set $\mathcal{N}_{x}$. For each agent, also called as a Voronoi site in the perspective of Voronoi diagram, there is a corresponding region consisting of all points closer to that agent than to any other. These regions are called Voronoi cells. The Voronoi cell, $V\left(a_{i}\right)$, associated with site $a_{i}$ is the set of all points in $X$ whose distances to $a_{i}$ are not greater than their distances to other sites $a_{j}, j \neq i$. In other words, if $d\left(x, a_{i}\right)$ denotes the distance between point $x$ and the site $a_{i}$, then

$$
V\left(a_{i}\right)=\left\{x \in X \mid d\left(x, a_{i}\right) \leq d\left(x, a_{j}\right) \text { for all } j \neq i\right\} .
$$

A target $\mathbf{t}$ is said to reside at agent $a_{i}$ if target $\mathbf{t}$ is closest to agent $a_{i}$ compared to all other agents in the monitoring area.

Agents in set $\mathcal{N}_{x}$ are classified into four kinds. Monitoring agent: An agent is called a monitoring agent if it is on one of the key positions that assures the area is fully 
covered. Tracking agent: An agent is called a tracking agent if it is tracking a target. Redundant agent: An agent is called a redundant agent if it is neither a monitoring nor tracking agent. Voronoi site agent: Monitoring agents, redundant agents, and the tracking agent in which the target resides are called Voronoi site agents.

Redundant agents are initially randomly pre-deployed in this area. However, monitoring agents are deployed in specific locations to achieve full coverage. To achieve full coverage of a specific 2-dimensional sensing field with an area $S_{c}$, ignoring boundary effects, the minimum number of agents $N_{m}$ required is calculated by equation (2) [17].

$$
N_{m}=\frac{S_{c}}{\pi\left(\frac{\sqrt{3} R_{s}}{2}\right)^{2}},
$$

where $R_{s}$ is the sensing radius of each agent.

The corresponding Voronoi diagram is shown in Fig. $1(a)$. A reasonable upper bound on the number of possible

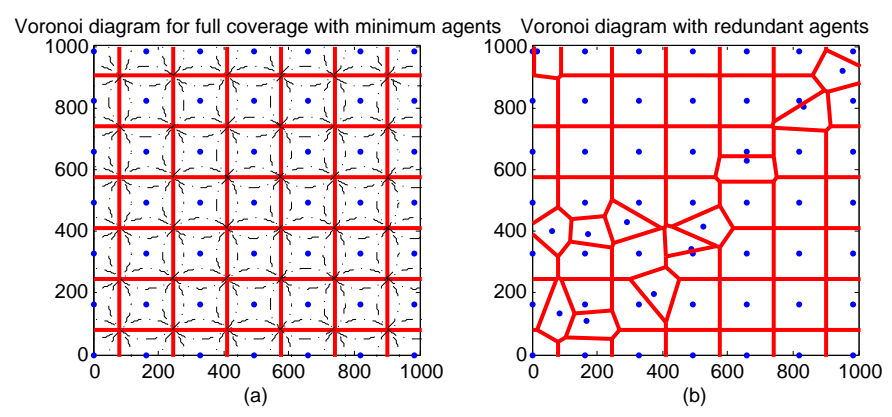

Fig. 1: Voronoi diagram for full coverage

targets can be defined as $N_{t}$ and each target is supposed to be tracked by a fixed number $\left(N_{f}\right)$ of tracking agents. Then the whole number of agents in this area can be easily calculated as $N_{a}=N_{m}+N_{t} N_{f}$. The Voronoi diagram with redundant agents deployed is shown in Fig. 1(b).

In Fig. 1(a), all the agents are monitoring agents. In Fig. 1(b), except the monitoring agents, the others randomly deployed are called redundant agents. All the agents in Fig. $1(b)$ can be called Voronoi site agents.

\section{B. Graph theory}

In the view of graph theory, each agent can be treated as a node. Then the communication topology of tracking agents and the target can be treated as a dynamic graph. A weighted graph $\mathrm{G}:=\{\mathcal{N}, \mathcal{E}, \mathcal{A}\}$ is denoted by a node set $\mathcal{N}=\left\{1,2, \ldots, N_{f}\right\}$, an edge set $\mathcal{E} \subseteq \mathcal{N} \times \mathcal{N}$ and a weighted adjacency matrix
$\mathcal{A}=\left[a_{i j}\right] \in \mathbb{R}^{N_{f} \times N_{f}}$ with nonnegative elements. An ordered edge of $\mathrm{G}$ is denoted by $(i, j)$, representing that agent $i$ is able to send information to agent $j$. If the graph is undirected, the edges $(i, j)$ and $(j, i)$ in $\mathcal{E}$ are considered to be the same. Throughout this paper, graph $\mathrm{G}$ is assumed to be undirected. The nodes within the communication range of node $i$ are called the set of neighbours of node $i$, which is denoted by $\mathcal{N}_{i}=$ $\{j \mid j \in \mathcal{N},(j, i) \in \mathcal{E}\}$. When $j \notin \mathcal{N}_{i}$, it is said node $j$ is beyond the communication range of node $i, a_{i j}=a_{j i}=0$, otherwise $a_{i j}=a_{j i}>0$. If $a_{i i} \neq 0$, we say that node has self-loop. In this paper, it is assumed that no self-loop exists. $b_{i}$ decides whether agent $i$ is able to communicate with the target or not. $b_{i}=0$ means agent $i$ cannot get the information of target, otherwise $b_{i}>0$. Denote $\mathcal{B}=\operatorname{diag}\left\{b_{1}, b_{2}, \ldots, b_{N_{f}}\right\}$.

The Laplacian matrix $\mathcal{L}=\left[l_{i j}\right] \in \mathbb{R}^{N_{f} \times N_{f}}$ of graph $\mathrm{G}$ is defined as $\mathcal{L}=\mathcal{D}-\mathcal{A}$, where

$$
\mathcal{D}=\operatorname{diag}\left\{\sum_{j \in \mathcal{N}_{1}} a_{1 j}, \sum_{j \in \mathcal{N}_{2}} a_{2 j}, \ldots, \sum_{j \in \mathcal{N}_{N_{f}}} a_{N_{f} j}\right\} .
$$

Since the dynamic change of the topology could happen when the target enters a new Voronoi cell, the Laplacian matrix is rewritten in the following format as $\mathcal{L}_{\sigma(t)}$, where $\sigma(t)=$ $k, t \in\left[t_{k}, t_{k+1}\right), k \in \mathbb{N}$.

Denote the communication graph by $\mathcal{G}=$ $\left\{G(0), G(1), G(2), \ldots, G\left(N_{s}\right)\right\}$, where $N_{s}$ is the total switching number and $G(k)=\left\{\mathcal{N}_{G(k)}, \mathcal{E}_{G(k)}, \mathcal{A}_{G(k)}\right\}, k \in \mathbb{N}$ is the communication graph. Denote the topology graph at time $t$ as $\mathcal{G}_{t}$, then $\mathcal{G}_{t}=G(k)$ when $\sigma(t)=k$. The switching topologies are event-triggered and then the topologies are piecewise constant between the event times $t_{0}, t_{1}, \ldots, t_{k}$.

At each event-triggered time, the tracking system may switch from a stable subsystem to an unstable subsystem or a stable subsystem, and vice versa. We introduce a sequence $p_{m}, m \in \mathbb{N}$, which belongs to the time sequence $t_{k}, k \in \mathbb{N}$, and the relationship between $t_{k}$ and $p_{m}$ satisfies

$$
\begin{aligned}
t_{0} & =p_{0}<t_{1}<\cdots<t_{s_{1}}=p_{1}<t_{s_{1}+1} \\
& <\cdots<t_{s_{2}}=<p_{2}<\cdots
\end{aligned}
$$

where $s_{m}, m \in \mathbb{N}$ is the number of switching among consecutive stable subsystems or consecutive unstable subsystems. $\bigcup_{m=0}^{+\infty}\left[p_{m}, p_{m+1}\right)=\left[t_{0},+\infty\right),\left[p_{m}, p_{m+1}\right)=\left[t_{s_{m}}, t_{s_{m}+1}\right) \cup$ $\left[t_{s_{m}+1}, t_{s_{m}+2}\right) \cup \cdots \cup\left[t_{s_{m+1}-1}, t_{s_{m+1}}\right)$. Then we suppose there exist a set of positive constants $\eta_{m}, \forall m \in \mathbb{N}$ for each interval of $\left[p_{m}, p_{m+1}\right)$, and a positive constant $\eta$ such that

$$
p_{m+1}-p_{m} \leq \eta_{m} \leq \eta<+\infty, \forall m \in \mathbb{N}
$$




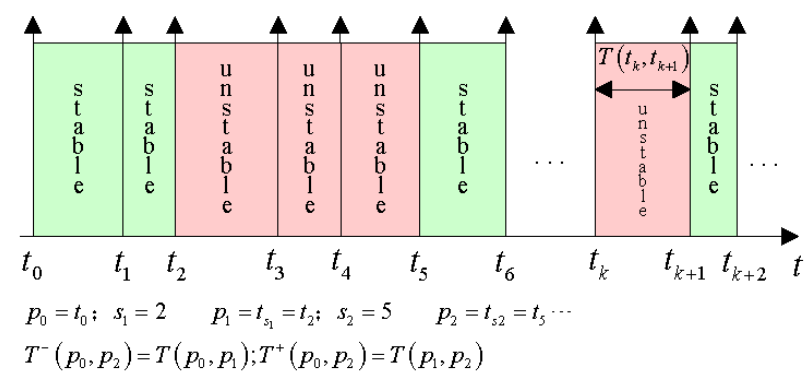

Fig. 2: Illustration of event-triggered switching times

The above paragraph can be interpreted as following. $t_{k}, k \in \mathbb{N}$ are all the switching moments including both switching between stable and unstable subsystems and switching from stable to stable or unstable to unstable subsystems. On the other hand, $p_{m}, m \in \mathbb{N}$ stand for the switching moments from a stable subsystem to an unstable subsystem or from an unstable subsystem to a stable subsystem. This is illustrated in Fig. 2.

$T\left(t_{k}, t_{k+1}\right)$ refers to the time interval $\left[t_{k}, t_{k+1}\right)$. For any time $\tau$ less than time $t, T^{+}(\tau, t)$ and $T^{-}(\tau, t)$ refer to the total time length of unstable and stable subsystems from initial time $\tau$ till the present time $t$, respectively. $l_{r}=\frac{T^{+}(\tau, t)}{T^{-}(\tau, t)}$ is called length rate of unstable subsystems on time interval $T(\tau, t)$. Let $N(\tau, t)$ denote the number of switching during $[\tau, t)$.

\section{Basic concepts}

Definition 1. [33] The system $\dot{x}(t)=f(t, x(t)), t \geq$ $t_{0}, x\left(t_{0}\right)=x_{0}, t_{0} \geq 0$ is globally exponentially stable if there exist positive scalars $c_{1}, c_{2}>0$ such that

$$
\|x(t)\| \leq c_{1}\left\|x_{0}\right\| e^{-c_{2}\left(t-t_{0}\right)}
$$

holds for all $t \geq t_{0}$.

Definition 2. [34] Let $x(t)$ be a solution of $\dot{x}(t)=$ $f(t, x(t)), t \geq t_{0}, x\left(t_{0}\right)=x_{0}, t_{0} \geq 0$ and $V(t, x(t))$ be a given function. Denote $D^{+} V(t, x(t))$ as the upper right-hand Dini derivative of $V(t, x(t))$, i.e.

$$
\begin{aligned}
& D^{+} V(t, x(t)) \\
= & \lim \sup _{h \rightarrow 0^{+}} \frac{V(t+h, x(t+h))-V(t, x(t))}{h} .
\end{aligned}
$$

Lemma 1. [35, 36]Given a graph $G$, then the Laplacian matrix $\mathcal{L}$ associated with the graph has at least one zero eigenvalue and all of the nonzero eigenvalues are in the open right half plane. For undirected graphs, the Laplacian matrix is symmetric with real eigenvalues. Therefore, the set of eigenvalues of $\mathcal{L}$ can be ordered sequentially in an ascending order as

$$
0=\lambda_{1}(\mathcal{L}) \leq \lambda_{2}(\mathcal{L}) \leq \ldots
$$

The eigenvalues of extended Laplacian matrix $\mathcal{L}+\mathcal{B}$ considering the target agent are all nonnegative. The mathematical statement of this is

$$
0 \leq \lambda_{i}(\mathcal{L}+\mathcal{B}), i \in \mathbb{N} .
$$

\section{PROBLEM FORMULATION AND RELAY TRACKING}

\section{ALGORITHM}

\section{A. Problem formulation}

The kinematic equation of the $i$-th agent deployed on the supervisory area is described as

$$
\begin{aligned}
& \dot{x}_{i}(t)=f\left(t, x_{i}(t)\right)+u_{i}(t), t \geq t_{0} \\
& x_{i}\left(t_{0}\right)=x_{i_{0}}, t_{0} \geq 0
\end{aligned}
$$

where $x_{i}(t) \in \mathbb{R}^{2}$ is position state of the $i$-th agent, $f\left(t, x_{i}(t)\right)$ is a nonlinear function, $u_{i}(t) \in \mathbb{R}^{2}$ is the control input of the $i$-th agent.

The agents are expected to monitor this area and capture any intruded target. We consider the following kinematic equations of the target.

$$
\begin{aligned}
& \dot{x}_{\mathbf{t}}(t)=f\left(t, x_{\mathbf{t}}(t)\right), t \geq t_{0} \\
& x_{\mathbf{t}}\left(t_{0}\right)=x_{\mathbf{t}_{0}}, t_{0} \geq 0
\end{aligned}
$$

where $x_{\mathbf{t}}(t) \in \mathbb{R}^{2}$ is position state of the target. $f\left(t, x_{\mathbf{t}}(t)\right)$ denotes the change of the force which is imposed on the target. In this paper, we assume $f(\cdot)$ satisfies the following constraint.

Assumption 1. There exists a nonnegative constant $l \geq 0$ such that

$$
\left\|f\left(t, x_{i}\right)-f\left(t, x_{\mathbf{t}}\right)\right\| \leq l\left\|x_{i}-x_{\mathbf{t}}\right\| .
$$

Assumption 1 is a Lipschitz-type condition, satisfied by many well-known systems including Lorenz system, Chen system, Lü system, Chua's circuit, and so on.

Definition 3. For first-order multi-agent tracking problem, tracking agents are supposed to move along with the target, i.e., the positions are required to be consensus. Mathematically, tracking problem boils down to the following expression.

$$
\lim _{t \rightarrow \infty}\left\|x_{i}(t)-x_{\mathbf{t}}(t)\right\| \rightarrow 0, i \in N_{f} .
$$

The purpose is to make sure positions of tracking agents and target finally to be the same. Thus, the following triggering 
event is adopted, which ensures the tracking errors decrease along with every switching.

$$
\begin{aligned}
& f_{e}=\left\|e_{x j}(t)\right\|-\left\|e_{x i}(t)\right\| \leq 0, j \neq i, \text { i.e. } \\
& d\left(x_{\mathbf{t}}(t), x_{j}(t)\right)=\left\|e_{x j}(t)\right\| \leq d\left(x_{\mathbf{t}}(t), x_{i}(t)\right)=\left\|e_{x i}(t)\right\|
\end{aligned}
$$

where $e_{x i}(t)=x_{i}(t)-x_{\mathbf{t}}(t)$ is the position disagreement vector between the $i$-th tracking agent and the target.

In this paper, $e_{x i}(t)=\left[e_{x_{i 1}}(t), e_{x_{i 2}}(t)\right]$ denotes the position disagreement vector, in which $e_{x_{i 1}}(t), e_{x_{i 2}}(t)$ are the position disagreement on $x-$ axis and $y$ - axis, respectively. Norm of the position disagreement vector between the $i$-th tracking agent and the target is the same as the distance between $i-$ th agent and the target, i.e., $\left\|e_{x i}(t)\right\|=$ $d\left(x_{\mathbf{t}}(t), x_{i}(t)\right)=\sqrt{e_{x_{i 1}}^{2}(t)+e_{x_{i 2}}^{2}(t)}$. Therefore, the triggering event (11) means that distance between agent $j$ and target is less than that of agent $i$. Then according to the definition of Voronoi diagram, this triggering event implies that the target moves from Voronoi cell $V\left(a_{i}\right)$ to Voronoi cell $V\left(a_{j}\right)$.

Remark 1. In this paper, the topology changes according to the movement of a target. When the target enters into a new Voronoi cell, the corresponding Voronoi site will become a tracking agent, meanwhile, one of the original tracking agents quits tracking. This means not only the topology changes but also the nodes, which is reflected by $\mathcal{N}_{G(k)}$. This is quite different from the traditional switched systems, in which only the topology switches.

\section{B. Relay tracking algorithm}

At each instant of time, only $N_{f}$ pursuers are assigned the task of tracking the maneuvering target, whereas all other agents in this region will remain stationary. The relay tracking algorithm is addressed as follows.

1) The essential number of agents for full coverage is calculated and the initial deployment of agents is formed in the interested area.

2) When a target enters the monitoring area, the Voronoi site agent and $N_{f}-1$ other nearest agents are set to be tracking agents to capture the intruded target.

3) In order to guarantee the full monitor of the whole area, the key position of each monitoring agent has to be occupied by at least one agent. Therefore, if the monitoring agent becomes a tracking agent, the redundant agent with shortest distance will shift to the key position of the original monitoring agent.
4) When the target escapes to one of the neighbour Voronoi cells. The new Voronoi site agent will transfer into a tracking agent. Meanwhile, the tracking agent with the furthest distance from the moving target will quit from the cooperative tracking process and 4) will repeat until the target is caught.

5) When the target is caught, it stops moving and releases the corresponding tracking agents to become redundant agents. This is called release policy. Repeat all the procedures until there is no more new and un-captured targets.

Remark 2. In this paper, every agent is able to communicate in two modes: omni and directional. In the omni mode, each agent is capable of receiving and transmitting signals in all directions (360 degrees) with gain $G$. On the other hand, in the directional mode, an agent can point its beam towards a specific direction with gain $G_{d}$ (where $G_{d}>G$ ). Because of the higher gain, agents in directional mode have a greater range in comparison with the omni mode [37].

At each time, it is assumed only the tracking agent, where the target resides, operates in directional mode while all the others operate in omni mode.

When a target enters the monitoring area, the corresponding Voronoi site agent $i$ operates in directional mode and rotates to send target's position to neighbouring agents. The neighbouring agents calculate their relative distances with the target and send this information back. Then, the Voronoi site agent $i$ decides the $N_{f}-1$ nearest agents and sends tracking commands to selected agents. Thereafter, Voronoi site agent stops rotating its communication direction and focuses on the direction of target.

Remark 3. Any changes in Voronoi diagram topology are solved in a local manner by means of well-known local algorithms [18], which means the moving of agents do not cause a global reassignment of Voronoi diagram.

According to the above analysis, computation complexity of the proposed relay pursuit tracking strategy is $O\left(2 N_{f}\right)$. It should be noted that this includes the tracking computation and dynamic Voronoi diagram computation.

\section{CONTROLLER DESIGN AND STABILITY ANALYSIS}

\section{A. Controller design}

It is assumed that agent $i$ can only receive the state information of its neighbours and target, then we adopt the 
following format of control protocol for the $i$-th tracking agent.

$$
u_{i}(t)=-\alpha\left\{\sum_{j \in \mathcal{N}_{i}(t)} a_{i j}(\sigma(t)) e_{x i j}(t)+b_{i}(\sigma(t)) e_{x i}(t)\right\}
$$

where $\alpha>0$ is the control parameter to be designed. $e_{x i j}(t)=$ $x_{i}(t)-x_{j}(t)$ is the position disagreement vector between the $i$-th tracking agent and the $j$-th tracking agent. Then, from Definition 3, the tracking problem can be interpreted as stability problem of the following overall disagreement system:

$$
\begin{aligned}
& \dot{\mathcal{E}}(t)=F(t, \mathcal{E}(t))-\alpha \tilde{\mathcal{L}}_{k} \mathcal{E}(t), t \neq t_{k} \\
& \mathcal{E}\left(t_{k}^{+}\right)=\mathcal{E}\left(t_{k}^{-}\right)-\Delta \mathcal{E}\left(t_{k}\right), t=t_{k}, k=0,1,2, \ldots
\end{aligned}
$$

where $\mathcal{E}(t)=\left[e_{x 1}^{T}(t), e_{x 2}^{T}(t), \ldots, e_{x N_{f}}^{T}(t)\right]^{T} \cdot \tilde{\mathcal{L}}_{k}=\left(\mathcal{L}_{k}+\right.$ $\left.\mathcal{B}_{k}\right) \otimes I_{2}$ is the associated Laplacian matrix of the dynamic graph including the target, in which $\mathcal{B}_{k}=$ $\operatorname{diag}\left\{b_{1}, b_{2}, \ldots, b_{\mathcal{N}_{f}}\right\}$ in time interval $\left[t_{k}, t_{k+1}\right) . F(t, \mathcal{E}(t))=$ $\operatorname{col}\left\{f_{1}(t), f_{2}(t), \ldots, f_{N_{f}}(t)\right\}$, where $f_{i}(t)=f\left(t, x_{i}(t)\right)-$ $f\left(t, x_{\mathbf{t}}(t)\right), i \in \mathcal{N}_{f}$ is the collective nonlinear self-dynamic disagreement to the target at time $t . t_{k}$ is the switching time. $t_{k}^{-}$indicates the time before switching and $t_{k}^{+}$represents the time after switching. $\Delta \mathcal{E}\left(t_{k}\right)$ is the jump of tracking error at switching time $t_{k}$.

Remark 4. As mentioned in Remark 1, when the topology switches, the tracking agent which is furthest to the target is replaced by the new Voronoi site. This means the norm of tracking error $\mathcal{E}(t)$ decreases at every switching time $t_{k}$. The element in $\mathcal{E}(t)$ corresponding to the replaced tracking agent jumps, and the jump value is reflected by $\Delta \mathcal{E}\left(t_{k}\right)$. For instance, if the $i-$ th tracking agent is replaced at time $t_{k}$, then $\left\|e_{x i}\left(t_{k}^{+}\right)\right\| \leq\left\|e_{x i}\left(t_{k}^{-}\right)\right\|$while $\left\|e_{x j}\left(t_{k}^{+}\right)\right\|=\left\|e_{x j}\left(t_{k}^{-}\right)\right\|, j \neq$ $i, i, j \in \mathcal{N}_{f}$. Tracking error jumps down at switching time $t_{k}$, which implies that $\left\|\mathcal{E}\left(t_{k}^{+}\right)\right\|^{2} \leq \mu\left\|\mathcal{E}\left(t_{k}^{-}\right)\right\|^{2}, 0<\mu \leq 1$.

Remark 5. In switched systems, sliding mode phenomenon arises when there are no impulse effects and the states do not jump at the switching events. However, in this paper, the states (tracking errors) jump due to our proposed relay pursuit strategy, in which a tracking agent is replaced by another one at the switching event. Therefore, the sliding mode phenomenon on the switching instants will not occur in the switched system with the proposed relay tracking strategy.

Next, we will analyze the multi-agent tracking system and figure out when it will be a stable subsystem or an unstable one. Let us select the Lyapunov candidate for the tracking system (13) during time interval $\left[t_{k}, t_{k+1}\right)$ as

$$
V(t)=\frac{1}{2} \mathcal{E}^{T}(t) \mathcal{E}(t), t \in\left[t_{k}, t_{k+1}\right), k \in \mathbb{N} .
$$

For any $t \in\left[t_{k}, t_{k+1}\right)$, the upper right-hand Dini derivative of $V(t)$ along the trajectory (13) is

$$
\begin{aligned}
D^{+} V(t) & =\frac{1}{2} \dot{\mathcal{E}}^{T}(t) \mathcal{E}(t)+\frac{1}{2} \mathcal{E}^{T}(t) \dot{\mathcal{E}}(t) \\
& =-\alpha \mathcal{E}^{T}(t) \tilde{\mathcal{L}}_{k}^{T} \mathcal{E}(t)+F(t, \mathcal{E}(t))^{T} \mathcal{E}(t) \\
& \leq\left(-\alpha \lambda_{\min }\left(\tilde{\mathcal{L}}_{k}\right)+l\right)\|\mathcal{E}(t)\|^{2}
\end{aligned}
$$

Apparently, with an event-triggered switching, the communication topologies change along with time, the tracking system can be stable or unstable. As stated in Lemma 1, the eigenvalues of Laplacian matrix are all nonnegative, as a result, there are two cases, i.e., $\lambda_{\min }\left(\tilde{\mathcal{L}}_{k}\right)>0$ and $\lambda_{\min }\left(\tilde{\mathcal{L}}_{k}\right)=0$. The corresponding two cases are addressed as following.

For some communication topologies, $\tilde{\mathcal{L}}_{k}$ is positive definite, we are always able to find appropriate positive control parameter $\alpha$ that enables $-\alpha \lambda_{\min }\left(\tilde{\mathcal{L}}_{k}\right)+l=-\gamma_{1}<0$, where $\gamma_{1}$ is a positive constant. In this case, we call the tracking system a stable subsystem. The corresponding Lyapunov function satisfies

$$
V(t)=V_{1}(t) \leq e^{-\gamma_{1}\left(t-t_{k}\right)} V\left(t_{k}\right)
$$

For some other communication topologies, $\lambda_{\min }\left(\tilde{\mathcal{L}}_{k}\right)=$ 0 , thus, $-\alpha \lambda_{\min }\left(\tilde{\mathcal{L}}_{k}\right)+l=\gamma_{2}>0$, where $\gamma_{2}$ is a positive constant. In this case, we call the tracking system an unstable subsystem. The corresponding Lyapunov function satisfies

$$
V(t)=V_{2}(t) \leq e^{\gamma_{2}\left(t-t_{k}\right)} V\left(t_{k}\right)
$$

It should be noted that the control parameter $\alpha$ is a constant and is determined off-line before the tracking process. According to the nonlinear dynamics of the agents, the Liptchitz coefficient can be calculated, and then it is able to obtain a conservative control parameter satisfying $-\alpha \lambda_{\min }\left(\tilde{\mathcal{L}}_{k}\right)+l=-\gamma_{1}<0$, where $\tilde{\mathcal{L}}_{k}$ is the worst possible case.

\section{B. Stability analysis}

Based on the above conditions, the overall stability of the tracking system is analyzed in this section.

Theorem 1. Consider the multi-agent system (13), if there exist positive numbers $\gamma^{*} \in\left(0, \gamma_{1}\right)$ and $\mu \leq 1$ such that the length rate $l_{r}$ of unstable subsystems satisfies

$$
l_{r}=\frac{T^{+}\left(t_{0}, t\right)}{T^{-}\left(t_{0}, t\right)} \leq \frac{\gamma_{1}-\gamma^{*}}{\gamma_{2}+\gamma^{*}},
$$


the tracking multi-agent system (13) with event-triggered switching topologies is overall stable. Moreover, the overall tracking error satisfies

$$
\|\mathcal{E}(t)\|^{2} \leq \mu^{N\left(t_{0}, t\right)}\left\|\mathcal{E}\left(t_{0}\right)\right\|^{2} e^{-\gamma^{*}\left(t-t_{0}\right)} .
$$

Proof. Without loss of generality, we assume, at the first time interval, the subsystem is stable. Then, during the time interval $\left[p_{0}, p_{1}\right)=\left[t_{0}, t_{s_{1}}\right)$, the Lyapunov function is

$$
\begin{aligned}
V_{1}\left(t_{1}^{-}\right)= & e^{-\gamma_{1} T\left(t_{0}, t_{1}\right)} V_{1}\left(t_{0}\right) \\
V_{1}\left(t_{1}^{+}\right) \leq & \mu V_{1}\left(t_{1}^{-}\right) \leq \mu e^{-\gamma_{1} T\left(t_{0}, t_{1}\right)} V_{1}\left(t_{0}\right) \\
V_{1}\left(t_{2}^{-}\right)= & e^{-\gamma_{1} T\left(t_{1}, t_{2}\right)} V_{1}\left(t_{1}^{+}\right) \\
V_{1}\left(t_{2}^{+}\right) \leq & \mu V_{1}\left(t_{2}^{-}\right) \leq \mu^{2} e^{-\gamma_{1} T\left(t_{0}, t_{2}\right)} V_{1}\left(t_{0}\right) \\
& \ldots \\
V_{1}\left(t_{s_{1}}^{-}\right)= & e^{-\gamma_{1} T\left(t_{s_{1}-1}, t_{s_{1}}\right)} V_{1}\left(t_{s_{1}-1}^{+}\right) .
\end{aligned}
$$

At time $p_{1}=t_{s_{1}}$, the tracking system switches from a stable subsystem to an unstable subsystem.

$$
\begin{aligned}
V_{2}\left(t_{s_{1}}^{+}\right) \leq & \mu V_{1}\left(t_{s_{1}}^{-}\right) \leq \mu^{s_{1}} e^{-\gamma_{1} T\left(t_{0}, t_{s_{1}}\right)} V_{1}\left(t_{0}\right) \\
V_{2}\left(t_{s_{1}+1}^{-}\right)= & e^{\gamma_{2} T\left(t_{s_{1}}, t_{s_{1}+1}\right)} V_{2}\left(t_{s_{1}}^{+}\right) \\
V_{2}\left(t_{s_{1}+1}^{+}\right) \leq & \mu V_{2}\left(t_{s_{1}+1}^{-}\right) \\
\leq & \mu^{s_{1}+1} e^{\gamma_{2} T\left(t_{s_{1}}, t_{s_{1}+1}\right)-\gamma_{1} T\left(t_{0}, t_{s_{1}}\right)} V_{1}\left(t_{0}\right) \\
& \cdots \\
V_{2}\left(t_{s_{2}}^{-}\right)= & e^{\gamma_{2} T\left(t_{s_{2}-1}, t_{s_{2}}\right)} V_{2}\left(t_{s_{2}-1}^{+}\right) .
\end{aligned}
$$

At time $p_{2}=t_{s_{2}}$, the tracking system switches from an unstable subsystem to a stable subsystem.

$$
\begin{aligned}
V_{1}\left(t_{s_{2}}^{+}\right) & \leq \mu V_{2}\left(t_{s_{2}}^{-}\right) \leq \mu^{s_{2}} e^{\gamma_{2} T\left(t_{s_{1}}, t_{s_{2}}\right)-\gamma_{1} T\left(t_{0}, t_{s_{1}}\right)} V_{1}\left(t_{0}\right) \\
V_{1}\left(t_{s_{2}+1}^{-}\right) & =e^{-\gamma_{1} T\left(t_{s_{2}}, t_{s_{2}+1}\right)} V_{1}\left(t_{s_{2}}^{+}\right) \\
V_{1}\left(t_{s_{2}+1}^{+}\right) & \leq \mu V_{1}\left(t_{s_{2}+1}^{-}\right) \\
& \leq \mu^{s_{2}+1} e^{\gamma_{2} T^{+}\left(t_{0}, t_{s_{2}+1}\right)-\gamma_{1} T^{-}\left(t_{0}, t_{s_{2}+1}\right)} V_{1}\left(t_{0}\right)
\end{aligned}
$$

Then, in general case, at time $t \in\left[t_{k}, t_{k+1}\right)$, the Lyapunov function boils down to

$$
V(t) \leq \mu^{k} e^{\gamma_{2} T^{+}\left(t_{0}, t\right)-\gamma_{1} T^{-}\left(t_{0}, t\right)} V\left(t_{0}\right) .
$$

From the condition (18) in Theorem 1, we can get

$$
\begin{aligned}
& T^{+}\left(t_{0}, t\right)\left(\gamma_{2}+\gamma^{*}\right)-T^{-}\left(t_{0}, t\right)\left(\gamma_{1}-\gamma^{*}\right) \leq 0 \\
& \gamma_{2} T^{+}\left(t_{0}, t\right)-\gamma_{1} T^{-}\left(t_{0}, t\right)+\gamma^{*}\left(T^{+}\left(t_{0}, t\right)+T^{-}\left(t_{0}, t\right)\right) \leq 0 \\
& \gamma_{2} T^{+}\left(t_{0}, t\right)-\gamma_{1} T^{-}\left(t_{0}, t\right) \leq-\gamma^{*}\left(t-t_{0}\right)
\end{aligned}
$$

Associate (23) with (24), it is straightforward to obtain that

$$
V(t) \leq \mu^{N\left(t_{0}, t\right)} V\left(t_{0}\right) e^{-\gamma^{*}\left(t-t_{0}\right)} .
$$

Since $V(t)=\frac{1}{2} \mathcal{E}^{T}(t) \mathcal{E}(t)=\frac{1}{2}\|\mathcal{E}(t)\|^{2}$ and $V\left(t_{0}\right)=$ $\frac{1}{2}\left\|\mathcal{E}\left(t_{0}\right)\right\|^{2}$. Then (19) follows from (25). According to Definition 1 , the overall error system is exponentially stable. The disagreement between the tracking agents and the target tends to 0 when $t \rightarrow \infty$. i.e., tracking agents finally track the target. Despite the system consists of unstable subsystems, the tracking agents still successfully track the target with the proposed tracking strategy.

\section{Disturbance attenuation Analysis}

In this section, we investigate the disturbance attenuation performance of our proposed tracking strategy for the multiagent system. The agents are subject to external disturbances, and the dynamic of the $i$-th agent is then described as

$$
\begin{aligned}
& \dot{x}_{i}(t)=f\left(t, x_{i}(t)\right)+u_{i}(t)+\omega_{i}(t), t \geq t_{0} \\
& x_{i}\left(t_{0}\right)=x_{i_{0}}, t_{0} \geq 0
\end{aligned}
$$

where $\omega_{i}(t) \in \mathscr{L}_{2}[0, \infty)$ is the disturbance input of the $i$-th agent.

The dynamic of the target is then described as

$$
\begin{aligned}
& \dot{x}_{\mathbf{t}}(t)=f\left(t, x_{\mathbf{t}}(t)\right)+\omega_{\mathbf{t}}(t), t \geq t_{0} \\
& x_{\mathbf{t}}\left(t_{0}\right)=x_{\mathbf{t}_{0}}, t_{0} \geq 0
\end{aligned}
$$

where $\omega_{\mathbf{t}}(t) \in \mathscr{L}_{2}[0, \infty)$ is the disturbance input of the target.

Then, from Definition 3, the tracking problem can be interpreted as stability problem of the following overall disagreement system:

$$
\begin{aligned}
& \dot{\mathcal{E}}(t)=F(t, \mathcal{E}(t))-\alpha \tilde{\mathcal{L}}_{k} \mathcal{E}(t)+\omega(t), t \neq t_{k} \\
& \mathcal{E}\left(t_{k}^{+}\right)=\mathcal{E}\left(t_{k}^{-}\right)-\Delta \mathcal{E}\left(t_{k}\right), t=t_{k}, k=0,1,2, \ldots
\end{aligned}
$$

where $\omega(t)=\left[\left(\omega_{1}(t)-\omega_{\mathbf{t}}(t)\right)^{T},\left(\omega_{2}(t)-\omega_{\mathbf{t}}(t)\right)^{T}, \ldots\right.$, $\left.\left(\omega_{N_{f}}(t)-\omega_{\mathbf{t}}(t)\right)^{T}\right]^{T}$.

For the limit of length and its similarity to the case without disturbance, some of the derivation is omitted.

For stable subsystems, there exists a positive scalar $\gamma_{1}^{\omega}$ satisfying $\frac{\gamma_{1}^{\omega}}{2} \leq \alpha \lambda_{\min }\left(\tilde{\mathcal{L}}_{k}\right)-l-\frac{\lambda_{0}^{-2}}{2}$, where $\lambda_{0}$ is a positive scalar positively involved with the disturbance attenuation level. The piecewise Lyapunov function $V(t)$ satisfies

$$
V(t) \leq e^{-\gamma_{1}^{\omega}\left(t-t_{k}\right)} V\left(t_{k}\right)+\frac{\lambda_{0}^{2}}{2} \int_{t_{k}}^{t} e^{-\gamma_{1}^{\omega}(t-\tau)} \omega^{T}(\tau) \omega(\tau) d \tau
$$

For unstable subsystems, $\lambda_{\min }\left(\tilde{\mathcal{L}}_{k}\right)=0$, there exists a positive scalar $\gamma_{2}^{\omega}$ satisfying $\frac{\gamma_{2}^{\omega}}{2} \geq l+\frac{\lambda_{0}^{-2}}{2}$, and then

$$
V(t) \leq e^{\gamma_{2}^{\omega}\left(t-t_{k}\right)} V\left(t_{k}\right)+\frac{\lambda_{0}^{2}}{2} \int_{t_{k}}^{t} e^{\gamma_{2}^{\omega}(t-\tau)} \omega^{T}(\tau) \omega(\tau) d \tau
$$


Now, we are ready to give the main result on the robustness of the overall tracking system, which states the disturbance attenuation level from $\omega$ to the tracking errors $\mathcal{E}$.

Theorem 2. Consider the disturbed multi-agent system (28), if there exist positive numbers $\gamma_{\omega}^{*} \in\left(0, \gamma_{1}^{\omega}\right)$ and $\mu \leq 1$ such that the length rate $l_{r}^{\omega}$ of unstable subsystems satisfies

$$
l_{r}^{\omega}=\frac{T^{+}\left(t_{0}, t\right)}{T^{-}\left(t_{0}, t\right)} \leq \frac{\gamma_{1}^{\omega}-\gamma_{\omega}^{*}}{\gamma_{2}^{\omega}+\gamma_{\omega}^{*}}
$$

the tracking multi-agent system (28) achieves a disturbance attenuation level $\frac{\lambda_{0}}{\sqrt{\gamma_{\omega}^{*}}}$.

Proof. According to (20)-(23), (31) and similar to the derivation of (24), we obtain

$$
V(t) \leq e^{-\gamma_{\omega}^{*}\left(t-t_{0}\right)} V\left(t_{0}\right)+\frac{\lambda_{0}^{2}}{2} \int_{t_{0}}^{t} e^{-\gamma_{\omega}^{*}(t-\tau)} \omega^{T}(\tau) \omega(\tau) d \tau
$$

By integrating the above inequality (32) in terms of time from $t_{0}$ to $\infty$, we have

$$
\begin{aligned}
\int_{t_{0}}^{\infty} V(t) d t \leq & \int_{t_{0}}^{\infty} e^{-\gamma_{\omega}^{*}\left(t-t_{0}\right)} V\left(t_{0}\right) d t+ \\
& \frac{\lambda_{0}^{2}}{2} \int_{t_{0}}^{\infty} \int_{t_{0}}^{t} e^{-\gamma_{\omega}^{*}(t-\tau)} \omega^{T}(\tau) \omega(\tau) d \tau d t \\
= & \frac{1}{\gamma_{\omega}^{*}} V\left(t_{0}\right)+\frac{\lambda_{0}^{2}}{2 \gamma_{\omega}^{*}} \int_{t_{0}}^{\infty} \omega^{T}(t) \omega(t) d t
\end{aligned}
$$

According to the definition of Lyapunov function $V(t)=$ $\frac{1}{2} \mathcal{E}^{T}(t) \mathcal{E}(t)$, the following inequality

$$
\int_{t_{0}}^{\infty} \mathcal{E}^{T}(t) \mathcal{E}(t) d t \leq \frac{2}{\gamma_{\omega}^{*}} V\left(t_{0}\right)+\frac{\lambda_{0}^{2}}{\gamma_{\omega}^{*}} \int_{t_{0}}^{\infty} \omega^{T}(t) \omega(t) d t
$$

holds for any $\omega(t) \in \mathscr{L}_{2}[0, \infty)$. This means that $\|\mathcal{E}\|_{\mathscr{L}_{2}}^{2} \leq$ $\frac{2}{\gamma_{\omega}^{*}} V\left(t_{0}\right)+\frac{\lambda_{0}^{2}}{\gamma_{\omega}^{*}}\|\omega\|_{\mathscr{L}_{2}}^{2}$, thus the disturbance attenuation level $\frac{\lambda_{0}}{\sqrt{\gamma_{\omega}^{*}}}$ is achieved. Our proposed tracking strategy is also robust against external disturbances for the multi-agent tracking systems.

\section{NUMERICAL EXAMPLES}

In this section we present numerical examples to show the effectiveness and correctness of the main results derived above. The numerical examples include the two cases where a target is tracked by three agents, and the case in which the agents are subject to disturbances. Both of the cases are conducted on the following scenario.
Consider a scenario where a group of three tracking agents track a maneuvering target in 2-dimensional space. The nonlinear dynamics of the tracking agents and target are

$$
\begin{aligned}
& f(t, x(t)) \\
= & 250 \sin (0.0035 x(t))+200 \cos (2.5 t)+20 \sin (2.5 t) .
\end{aligned}
$$

Then, $\left\|f\left(t, x_{i}\right)-f\left(t, x_{\mathbf{t}}\right)\right\| \leq 250 \times 0.0035\left\|x_{i}-x_{\mathbf{t}}\right\|=$ $0.875\left\|x_{i}-x_{\mathbf{t}}\right\|$. Consider there are at most 3 targets may enter a $1000 \times 1000 \mathrm{~m}^{2}$ monitoring area and the sensing radius is set as $R_{s}=125 \mathrm{~m}$. The time of the first target breaks into this domain is considered as initial time. The coordinate where the first target enters this area is $(0,80)$.

\section{A. The target is tracked by three agents}

This example addresses the situation in which an intruded target is tracked by three agents. The tracking process is illustrated in Fig. 3. In this figure, red line represents the trajectory of target. When the target enters a new Voronoi cell, the associated Voronoi site agent replaces one of the original tracking agents, which leads the tracking trajectories to be discontinuous. The discontinuity of different colored lines clearly shows which agent is replaced.

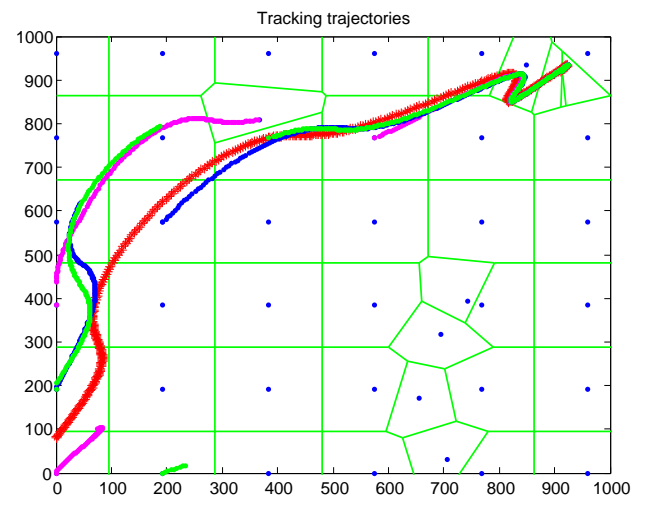

Fig. 3: Tracking trajectories

Fig. 4 shows tracking errors on $x$-axis, $y$-axis, which obviously reflects the switching. Every switching of topology and tracking agents brings a jump in tracking error. Actually, each discontinuity of a line in Fig. 3 corresponds to a jump in Fig. 4. For instance, at step 158, agent 3 is replaced by a new Voronoi site agent and its error trajectory of $x$ - axis sees a significant jump. Correspondingly, there is an obvious discontinuity in the green line as wee can see in Fig. 3.

Fig. 5 depicts the switching signal $\sigma(t)$. The system switches five times over the entire tracking process, which is verified by the summation of norm of all tracking errors, i.e., 

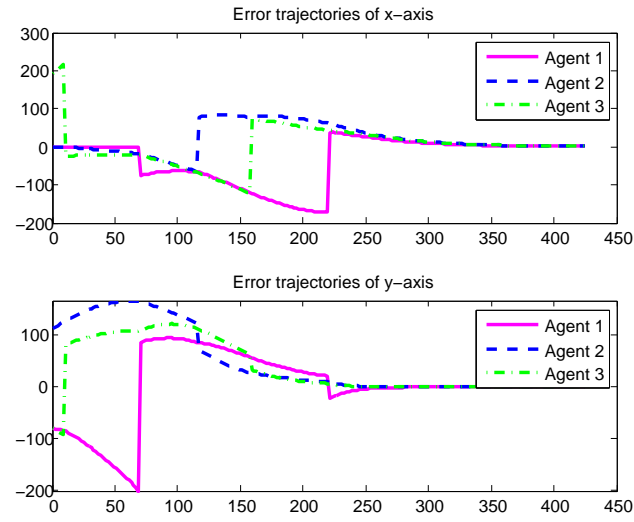

Fig. 4: Tracking errors of three tracking agents

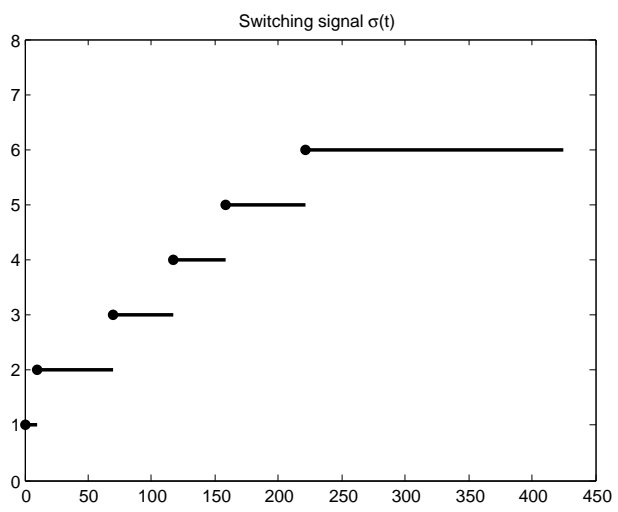

Fig. 5: Switching signal of the tracking process
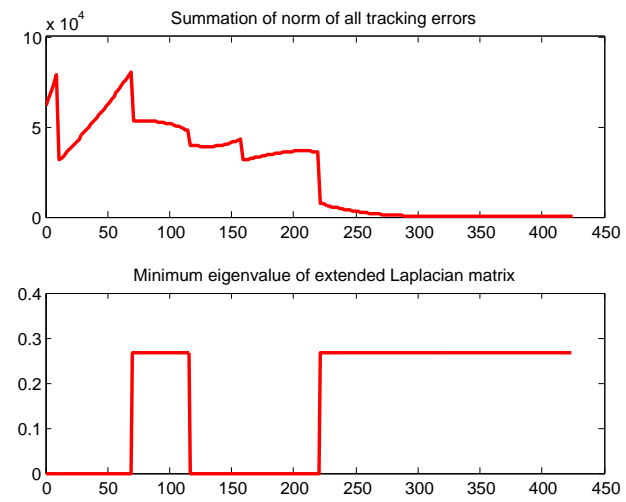

Fig. 6: Norm of errors and minimum eigenvalues

the value of Lyapunov function in Fig. 6. At every switching, the summation of norm of all tracking errors jumps down, satisfying $V\left(t_{k+1}\right) \leq \mu V\left(t_{k}\right)$.

According to Fig. 6, during simulation steps $(0,69)$ and $(117,220)$, the minimum eigenvalues of extended Laplacian matrix is 0 , i.e., $\lambda_{\min }\left(\tilde{\mathcal{L}}_{k}\right)=0$, then $-\alpha \lambda_{\text {min }}\left(\tilde{\mathcal{L}}_{k}\right)+l=$ $\gamma_{2}=0.875$, the related subsystems are unstable subsystems and the corresponding Lyapunov function is increasing as can be clearly seen from Fig. 6. At the other time steps $\lambda_{\text {min }}\left(\tilde{\mathcal{L}}_{k}\right)=0.2679$. To ensure the stability of the subsystems, it is required $-\alpha \lambda_{\min }\left(\tilde{\mathcal{L}}_{k}\right)+l<0$, then the designed control parameter is $\alpha>3.2661$. Under this design principle, we choose the control parameter as $\alpha=6$, thus $\gamma_{1}=0.7324$. Thus, the summation norm of tracking error holds a decreasing trend. Select $\gamma^{*}=0.3$, we have $\frac{\gamma_{1}-\gamma^{*}}{\gamma_{2}+\gamma^{*}}=0.4942$, and then $l_{r}=\frac{T^{+}\left(t_{0}, t\right)}{T^{-}\left(t_{0}, t\right)}=0.2767<\frac{\gamma_{1}-\gamma^{*}}{\gamma_{2}+\gamma^{*}}$. It is verified that the condition in Theorem 1 is satisfied.

\section{B. The agents are subject to disturbances}

A simulation of multi-agent tracking system with disturbances is conducted to verify the theoretical result in Section V. For simulation analysis, we assume a disturbance $d(t)$ shown in Fig. 7, and the disturbances imposed on target and three tracking agents are $\omega_{\mathbf{t}}(t)=d(t), \omega_{1}(t)=$ $1.2 d(t), \omega_{2}(t)=0.5 d(t)$ and $\omega_{3}(t)=-d(t)$ respectively. The rest of the simulation scenario is the same as that in Example VI-A. The tracking trajectories of the disturbed system is shown in Fig. 8.

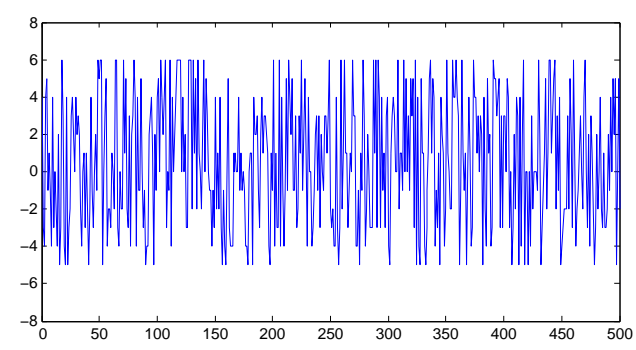

Fig. 7: Illustrative description of disturbance signal $d(t)$

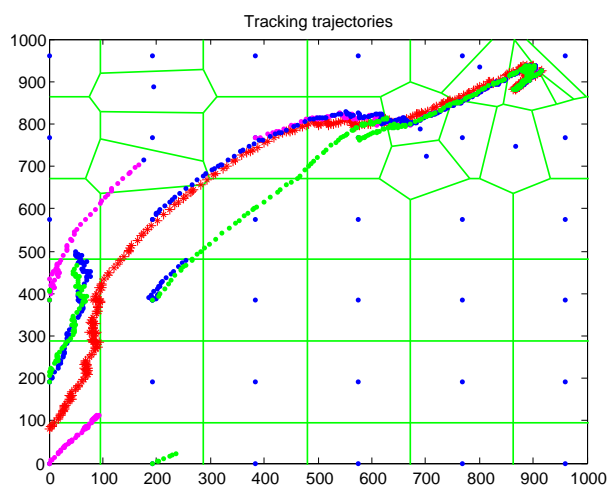

Fig. 8: Tracking trajectories of the system subject to disturbances

Choose $\lambda_{0}=6$, we obtain $\gamma_{1}^{\omega}=1.3, \gamma_{2}^{\omega}=1.9$. Select $\gamma_{\omega}^{*}=0.2$, we have $\frac{\gamma_{1}^{\omega}-\gamma_{\omega}^{*}}{\gamma_{2}^{\omega}+\gamma_{\omega}^{*}}=0.5238$. In this example, 


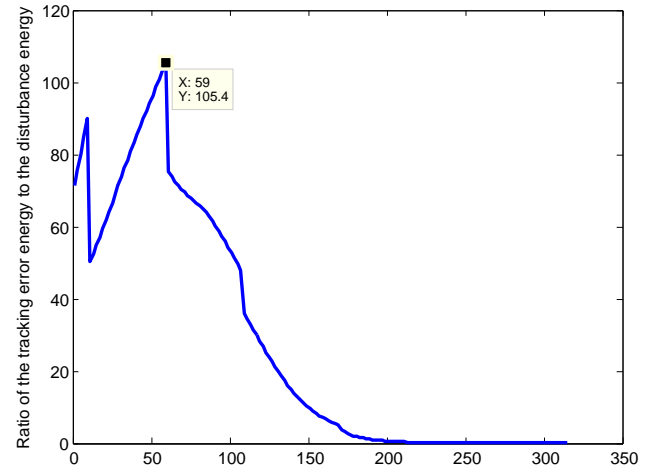

Fig. 9: Ratio of the tracking error energy to the disturbance energy

the length rate of unstable subsystems is $l_{r}=0.4959$, which apparently satisfies the condition in Theorem 2 . The disturbance attenuation level is designed as $\frac{\lambda_{0}}{\sqrt{\gamma_{\omega}^{*}}}=13.4164$. The ratio of the tracking error energy to the disturbance input energy, that is, $\int_{t_{0}}^{\infty} \mathcal{E}^{T}(t) \mathcal{E}(t) d t / \int_{t_{0}}^{\infty} \omega^{T}(t) \omega(t) d t$ is depicted in Fig. 9, which shows that the maximum value of the ratio is 105.4. Therefore, the disturbance attenuation level is $\sqrt{105.4}=10.2665$, which verifies that the designed value $\frac{\lambda_{0}}{\sqrt{\gamma_{\omega}^{*}}}=13.4164$ is achieved.

\section{Comparison of tracking performance with non-relay method}

To verify the advantage of proposed tracking strategy, we compare the tracking performance between the relay pursuit strategy and the non-relay pursuit strategy. In the two tracking strategies, the initial tracking agents are the same. However, in the non-relay pursuit strategy, the tracking agents do not switch when the target enters a new Voronoi cell.

Fig. 10 shows norms of tracking errors with two tracking strategies. As can be seen from the figure, it is obvious that the target can be tracked successfully within a shorter time with proposed tracking strategy. The norms of tracking errors at steps 200 and 300 are marked. Specifically, at step 300, the norm of tracking errors with proposed strategy is 430.4 , which is overwhelmingly less than its counterpart value 11130 of the non-relay strategy.

\section{CONCLUSIONS}

The tracking problem of a class of nonlinear multi-agent systems on a certain domain is investigated. This domain is monitored by a number of smart agents and divided into many
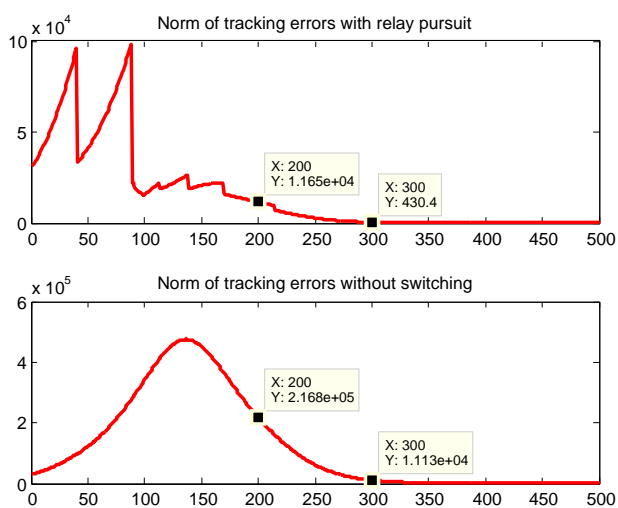

Fig. 10: Norm of tracking errors comparison

Voronoi cells with assistance of Voronoi diagram. At the same time, the smart agents are set to track any intruded target agent with the proposed tracking strategy. Several tracking agents cooperate and communicate with each other when they are supposed to track the same target. During the tracking process, the tracking agents are relayed by a series of Voronoi sites. The switching of topologies and relay of tracking agents are triggered when a target is moving into a new Voronoi cell and this leads the tracking system to be stable or unstable subsystems. The system not only switches among consecutive stable subsystems or consecutive unstable subsystems but also between stable and unstable subsystems. Our proposed tracking strategy guarantees overall successful tracking with tolerance of unstable subsystems and disturbances.

One issue that has not been addressed in this paper, and is worth pursuing in the future work, is whether this cooperative relay tracking strategy can be used to capture a target under the network-based environment with time-delays and packet dropouts, which is known to be an important issue in networked systems $[38,39]$. In this case, the subsystems are more likely to be unstable and it will be more difficult to deal with. Another possible extension is to consider a situation where the agents and targets are described by TakagiSugeno (T-S) fuzzy dynamic models [40-43]. In terms of more complex nonlinear systems, when the state variable is not measurable, observer-based control $[44,45]$ could be adopted.

\section{REFERENCES}

[1] T. Vicsek, "A question of scale," Nature, vol. 411, no. 6836, pp. 421-421, 2001.

[2] J. Qin, H. Gao, and W. X. Zheng, "Exponential synchronization of complex networks of linear systems and non- 
linear oscillators: A unified analysis," Neural Networks and Learning Systems, IEEE Transactions on, vol. 26, no. 3, pp. 510-521, March 2015.

[3] J. Qin and C. Yu, "Coordination of multiagents interacting under independent position and velocity topologies," Neural Networks and Learning Systems, IEEE Transactions on, vol. 24, no. 10, pp. 1588-1597, Oct 2013.

[4] Z. Meng, Z. Li, A. Vasilakos, and S. Chen, "Delayinduced synchronization of identical linear multiagent systems," IEEE Transactions on Cybernetics, vol. 43, no. 2, pp. 476-489, 2013.

[5] Y. Tang, H. Gao, W. Zou, and J. Kurths, "Distributed synchronization in networks of agent systems with nonlinearities and random switchings," IEEE Transactions on Cybernetics, vol. 43, no. 1, pp. 358-370, 2013.

[6] W. Wang, J. Huang, C. Wen, and H. Fan, "Distributed adaptive control for consensus tracking with application to formation control of nonholonomic mobile robots," Automatica, vol. 50, no. 4, pp. 1254-1263, 2014.

[7] P. Wang and B. Ding, "Distributed rhc for tracking and formation of nonholonomic multi-vehicle systems," IEEE Transactions on Automatic Control, vol. 59, no. 6, pp. 1439-1453, 2014.

[8] A. Yang, W. Naeem, G. W. Irwin, and K. Li, "Stability analysis and implementation of a decentralized formation control strategy for unmanned vehicles," IEEE Transactions on Control Systems Technology, vol. 22, no. 2, pp. 706-720, 2014.

[9] Y. Jiang, J. Liu, and S. Wang, "Consensus tracking algorithm via observer-based distributed output feedback for multi-agent systems under switching topology," Circuits Systems and Signal Processing, vol. 33, no. 10, pp. 30373052, 2014.

[10] G. Wen, Z. Duan, G. Chen, and W. Yu, "Consensus tracking of multi-agent systems with lipschitz-type node dynamics and switching topologies," IEEE Transactions on Circuits and Systems I-Regular Papers, vol. 61, no. 2, pp. 499-511, 2014.

[11] L. Dong, S. Chai, and B. Zhang, "Tracking of a thirdorder maneuvering target under an arbitrary topology," Chin. Phys. B, vol. 23, no. 1, p. 010508, 2014.

[12] L. Dong, S. Chai, B. Zhang, and S. Nguang, "Tracking problem under a time-varying topology," Chin. Phys. B, vol. 23, no. 6, p. 060502, 2014.
[13] L. Dong, S. Chai, B. Zhang, and S. K. Nguang, "Sliding mode control for multi-agent systems under a time-varying topology," International Journal of Systems Science, vol. 47, no. 9, pp. 2193-2200, 2016.

[14] L. Dong, S. Chai, B. Zhang, S. K. Nguang, and L. Wen, "High-order tracking problem with a time-varying topology and communication delays," Neurocomputing, vol. 149, Part C, no. 0, pp. 1360 - 1369, 2015.

[15] E. Bakolas and P. Tsiotras, "Relay pursuit of a maneuvering target using dynamic voronoi diagrams," Automatica, vol. 48, no. 9, pp. 2213-2220, 2012.

[16] E. Bakolas, P. Tsiotras, and IEEE, “Optimal pursuit of moving targets using dynamic voronoi diagrams," 49th IEEE Conference on Decision and Control (CDC), pp. 7431-7436, 2010.

[17] J. S. Li and H. C. Kao, "Distributed k-coverage selflocation estimation scheme based on voronoi diagram," IET Communications, vol. 4, no. 2, pp. 167-177, 2010.

[18] M. A. Mostafavi, C. Gold, and M. Dakowicz, "Delete and insert operations in voronoi/delaunay methods and applications," Computers and Geosciences, vol. 29, no. 4, pp. 523-530, 2003.

[19] O. Hajek, Pursuit games: an introduction to the theory and applications of differential games of pursuit and evasion. Mineola, New York: Dover Publications, 2008.

[20] Y. Zhang and Y. Meng, "A decentralized multi-robot system for intruder detection in security defense," in Intelligent Robots and Systems (IROS), 2010 IEEE/RSJ International Conference on, Oct 2010, pp. 5563-5568.

[21] Y. Xiao and Y. Zhang, "Surveillance and tracking system with collaboration of robots, sensor nodes, and rfid tags," in Computer Communications and Networks, 2009. ICCCN 2009. Proceedings of 18th Internatonal Conference on, Aug 2009, pp. 1-6.

[22] X. Sun and W. Wang, "Integral input-to-state stability for hybrid delayed systems with unstable continuous dynamics," Automatica, vol. 48, no. 9, pp. 2359-2364, 2012.

[23] X. Sun, S. Du, P. Shi, W. Wang, and L. Wang, "Input-tostate stability for nonlinear systems with large delay periods based on switching techniques," IEEE Transactions on Circuits and Systems, vol. 61, no. 6, pp. 1789-1800, 2014.

[24] J. Qin, C. Yu, and H. Gao, "Coordination for linear 
multiagent systems with dynamic interaction topology in the leader-following framework," Industrial Electronics, IEEE Transactions on, vol. 61, no. 5, pp. 2412-2422, May 2014.

[25] J. Qin, H. Gao, and C. Yu, "On discrete-time convergence for general linear multi-agent systems under dynamic topology," Automatic Control, IEEE Transactions on, vol. 59, no. 4, pp. 1054-1059, April 2014.

[26] S. Saat and S. K. Nguang, "Nonlinear $\mathrm{H} \infty$ output feedback control with integrator for polynomial discrete-time systems," International Journal of Robust and Nonlinear Control, vol. 25, no. 7, pp. 1051-1065, 2015.

[27] S. Chae and S. K. Nguang, "SOS based robust H $\infty$ fuzzy dynamic output feedback control of nonlinear networked control systems," IEEE Transactions on Cybernetics, vol. 44, no. 7, pp. 1204-1213, 2014.

[28] X. Wang, S. Li, and P. Shi, "Distributed finite-time containment control for double-integrator multiagent systems," IEEE Transactions on Cybernetics, vol. 44, no. 9, pp. 1518-1528, 2014.

[29] Y. Liu and J. Lunze, "Leader-follower synchronisation of autonomous agents with external disturbances," International Journal of Control, vol. 87, no. 9, pp. 1914-1925, 2014.

[30] Y. Zhao, B. Li, J. Qin, H. Gao, and H. R. Karimi, " $\mathrm{H} \infty$ consensus and synchronization of nonlinear systems based on a novel fuzzy model," IEEE Transactions on Cybernetics, vol. 43, no. 6, pp. 2157-2169, 2013.

[31] D. Meng and K. Moore, "Studies on resilient control through multiagent consensus networks subject to disturbances," IEEE Transactions on Cybernetics, vol. 44, no. 11, pp. 2050-2064, 2014.

[32] I. Saboori and K. Khorasani, "Hळ consensus achievement of multi-agent systems with directed and switching topology networks," IEEE Transactions on Automatic Control, vol. 59, no. 11, pp. 3104-3109, 2014.

[33] P. H. A. Ngoc, "New criteria for exponential stability of nonlinear time-varying differential systems," International Journal of Robust and Nonlinear Control, vol. 24, no. 2, pp. 264-275, 2014.

[34] N. M. Linh and V. N. Phat, "Exponential stability of nonlinear time-varying differential equations and applications," Electronic Journal of Differential Equations, vol. 2001, no. 34, pp. 1-13, 2001.
[35] W. Ren and R. W. Beard, "Consensus seeking in multiagent systems under dynamically changing interaction topologies," IEEE Transactions on Automatic Control, vol. 50, no. 5, pp. 655-661, 2005.

[36] R. Olfati-Saber, J. Fax, and R. Murray, "Consensus and cooperation in networked multi-agent systems," Proceedings of the IEEE, vol. 95, no. 1, pp. 215-233, 2007.

[37] V. Friderikos, K. Papadaki, M. Dohler, A. Gkelias, and H. Agvhami, "Linked waters [marine communication]," Communications Engineer, vol. 3, no. 2, pp. 24-27, 2005.

[38] H. Li, C. Wu, P. Shi, and Y. Gao, "Control of nonlinear networked systems with packet dropouts: Interval type2 fuzzy model-based approach," IEEE Transactions on Cybernetics, vol. 45, no. 11, pp. 2378-2389, 2014.

[39] W. Tong, H. Gao, and J. Qiu, "A combined adaptive neural network and nonlinear model predictive control for multirate networked industrial process control," IEEE Transactions on Neural Networks and Learning Systems, vol. 27, no. 99, pp. 416-425, 2015.

[40] J. Qiu, S. Ding, H. Gao, and S. Yin, "Fuzzy-modelbased reliable static output feedback $\mathrm{H} \infty$ control of nonlinear hyperbolic pde systems," Fuzzy Systems, IEEE Transactions on, vol. PP, no. 99, pp. 1-13, 2015.

[41] J. Qiu, G. Feng, and H. Gao, "Static-output-feedback $\mathrm{H} \infty$ control of continuous-time T-S fuzzy affine systems via piecewise lyapunov functions," Fuzzy Systems, IEEE Transactions on, vol. 21, no. 2, pp. 245-261, April 2013.

[42] J. Qiu, H. Tian, Q. Lu, and H. Gao, "Nonsynchronized robust filtering design for continuous-time T-S fuzzy affine dynamic systems based on piecewise lyapunov functions," Cybernetics, IEEE Transactions on, vol. 43, no. 6, pp. 1755-1766, 2013.

[43] J. Zhang, P. Shi, J. Qiu, and S. K. Nguang, "A novel observer-based output feedback controller design for discrete-time fuzzy systems," IEEE Transactions on Fuzzy Systems, vol. 23, no. 1, pp. 223-229, 2015.

[44] H. Li, Y. Gao, P. Shi, and H. Lam, "Observer-based fault detection for nonlinear systems with sensor fault and limited communication capacity," IEEE Transactions on Automatic Control, pp. 1-1, 2015.

[45] H. Li, C. Wu, S. Yin, and H. Lam, "Observer-based fuzzy control for nonlinear networked systems under unmeasurable premise variables," IEEE Transactions on Fuzzy Systems, pp. 1-1, 2015. 
2016-06-30

\section{Stability of a class of multi-agent tracking systems with unstable subsystems}

Dong, Lijing

IEEE

Dong L, Chai S, Zhang B, Nguang SK, Savvaris A, Stability of a class of multi-agent tracking systems with unstable subsystems, IEEE Transactions on Cybernetics, Vol. 47, Issue 8, August 2017, pp. 2193-2202

http://dx.doi.org/10.1109/TCYB.2016.2581830

Downloaded from Cranfield Library Services E-Repository 